Suisse. Professionnels, oui. Mais intra muros.

Études de cas

Danièle Périsset-Bagnoud

\title{
CpenEdition
}

Journals

Édition électronique

URL : https://journals.openedition.org/ries/1307

DOI : $10.4000 /$ ries. 1307

ISSN : 2261-4265

Éditeur

France Education international

Édition imprimée

Date de publication : 1 décembre 2005

Pagination : 94-96

ISSN : 1254-4590

Référence électronique

Danièle Périsset-Bagnoud, « Suisse. Professionnels, oui. Mais intra muros. », Revue internationale d'éducation de Sèvres [En ligne], 40 | décembre 2005, mis en ligne le 17 novembre 2011, consulté le 05 juillet 2021. URL : http://journals.openedition.org/ries/1307; DOI : https://doi.org/10.4000/ries.1307

Ce document a été généré automatiquement le 5 juillet 2021.

(c) Tous droits réservés 


\section{Suisse. Professionnels, oui. Mais intra muros.}

Études de cas

Danièle Périsset-Bagnoud

1 Plusieurs initiatives ont tenté, et tentent encore, de transformer le système d'éducation suisse et, notamment, de faire avancer le processus de professionnalisation de l'enseignement. Plusieurs catégories d'acteurs y participent : les acteurs politiques, qui ont proposé, au cours de ces dix dernières années, différents cadres législatifs propres à favoriser notamment l'accroissement de l'autonomie des établissements et à inscrire les plans d'étude dans une perspective longitudinale inédite, les acteurs professionnels, par leurs associations impliquées dans les réformes politiques; les acteurs de la recherche en éducation, qui fournissent aux précédents des fondements scientifiques sur lesquels se basent les propositions de réformes.

\section{De la professionnalisation}

2 Cependant, si l'on trouve, à l'intérieur même de chacune de ces catégories d'acteurs, diverses tendances qui nourrissent bien des paradoxes autour de l'objet "professionnalisation de l'enseignement », les positions que prennent les enseignants, individuellement ou par leurs associations, ne sont pas les moins étonnantes.

3 Le terme de professionnalisation est aujourd'hui couramment utilisé. Pourtant, il suscite bien des débats. Ces qualités font cependant consensus: l'enseignant professionnel est au bénéfice d'une formation, longue et spécialisée, de niveau supérieur et qui relève de savoirs scientifiques; il réalise en autonomie des actes intellectuels et non routiniers qui engagent sa responsabilité; le corps professionnel est structuré en organisme officiel auto-régulé et fort d'une reconnaissance sociale élevée.

4 C'est la seconde caractéristique qui retient à ce jour le plus l'attention des associations d'enseignants. Autonomie et responsabilité sont les maîtres mots des discours relevant 
des évolutions des systèmes scolaires et se déclinent sur toute la gamme de leurs possibles interprétations. Paradoxes et ambiguïtés nourrissent les positions des enseignants que révèlent deux documents récents : le Code de déontologie du Syndicat des enseignants romands (2004) et les résultats d'une enquête menée en 2002 auprès de onze mille enseignants en Suisse alémanique par leur association professionnelle (LCH).

\section{Autonomes et responsables, mais dans les murs de la classe}

5 Une lecture attentive des déclarations d'intention du Code de déontologie révèle la trame des difficultés. Ainsi est souhaitée une lecture critique et responsable de ce à quoi est exposé l'enseignant ( « faire preuve de sens critique, d'autonomie et savoir prendre ses responsabilités»). Aucune trace de collaboration/partenariat/négociation éventuels avec la hiérarchie ou les pairs n'apparaît. À quel implicite, et à qui, rattacher ce sens critique, sinon à celui que peut mettre chaque acteur, d'après ses expériences et stratégies personnelles? Pourtant, point trop de liberté ne faut: un bon professionnel tient « compte des options pédagogiques de son association professionnelle». Plus loin, une norme intériorisée, puisque non définie, est donnée pour évidente et partagée : «faire preuve de conscience professionnelle ». Cette terminologie désuète trahit la persistance de l'origine normalienne des enseignants. La professionnalisation donnée à voir ici reste confinée dans les murs de la classe, s'y gonfle d'une autonomie toute relative et ne s'aventure guère au dehors, où sévit un environnement public, politique, dangereux.

6 L'enquête individuelle auprès des enseignants confirme ce sentiment de repli. Elle révèle la permanence du lieu commun lié à la nostalgie d'un passé mythique d'avant la démocratisation-massification des études, lorsque les publics étaient clairement sélectionnés selon leur provenance sociale et ne posaient, de ce point de vue, guère de problème. L'environnement social et éducatif est vu comme déficitaire ; les initiatives de la hiérarchie sont rejetées (excès de réformes et absence de consultation) mais, le paradoxe n'est pas le moindre, le manque de soutien et de directives de la part de ces mêmes autorités est déploré. L'individu s'adapte apparemment aux changements mais dans son for intérieur, il se rebelle et en appelle à l'ancien ordre social. L'enseignant veut être professionnel, certes. Mais dans le cadre de sa classe, dans son jardin protégé et sous le regard « bienveillant » et paternel d'une hiérarchie qui le guide.

\section{Acteurs, mais insoumis}

7 Entre les intentions déclarées des systèmes éducatifs et celles qui leur sont prêtées, entre les stratégies des associations d'enseignants et celles des enseignants, il y a tout un jeu de stratégies que nourrissent les enjeux spécifiques des différents groupes d'acteurs et que sous-tendent les cultures collectives issues de l'histoire longue des mentalités corporatives. Leur identité ne se modifie pas par décret. La transformation des systèmes éducatifs accompagne étroitement le développement social, qui marque lui-même l'évolution des formations d'enseignants. L'histoire de l'école, en perpétuelle évolution, est faite de tensions et de ruptures, de permanences, de tentatives 
de changements et de résistances. C'est une évidence souvent oubliée. Et l'évolution est mue par les conflits entre les groupes d'acteurs sociaux.

8 Les enseignants, assurément, sont acteurs de l'évolution des systèmes scolaires, mais dans «leur " sens, celui qui répond le mieux à leurs besoins, à leurs stratégies. Leur apparente résistance est peut-être le moyen qu'ils privilégient pour dire leur difficulté à entrer dans un jeu d'enjeux dans lesquels ils ne se reconnaissent pas mais dans lequel ils voudraient qu'on leur donne la place, ultime paradoxe, qu'ils ne savent, ne peuvent, ne veulent peut-être pas encore conquérir.

INDEX

Index géographique : Suisse

\section{AUTEUR}

DANIĖLE PÉRISSET-BAGNOUD

Professeur, Haute école pédagogique du Valais et Université de Genève. 